\title{
Sjögren-Larsson Syndrome: A Case Report of a Rare Neuro-cutaneous Disorder
}

\author{
Manish Pradhan ${ }^{1}$, Arun Giri' ${ }^{2}$ Dipa Rai ${ }^{1}$, Rajeev Yadav ${ }^{l}$ \\ 'Department of Dermatology, Venereology and Leprosy, Nobel Medical College Teaching Hospital, Biratnagar, Nepal \\ ${ }^{2}$ Department of Pediatrics, Nobel Medical College Teaching Hospital, Biratnagar, Nepal
}

\section{ABSTRACT}

Sjögren-Larsson syndrome is a rare autosomal recessive neuro-cutaneous disease. The dominant features are congenital ichthyotic hyperkeratosis, spastic diplegia and mild to moderate mental retardation. The cause of this syndrome is the deficiency of microsomal fatty aldehyde dehydrogenase. The deficiency of this enzyme results in the accumulation of fatty aldehydes and fatty alcohols in various tissues including skin. Here, we report a case presented with ichthyosis, bilateral lower limb spasticity and mental retardation, diagnosed as Sjögren-Larsson syndrome.

Keywords: : Ichthyosis; Mental retardation; Spastic diplegia

\author{
Correspondence: \\ Dr. Manisha Pradhan, MD \\ Department of Dermatology, Venereology and \\ Leprosy, \\ Nobel Medical College Teaching Hospital, \\ Biratnagar, Nepal \\ ORCID ID: 0000-0001-6817-4160 \\ Email: drmanishpradhan1@gmail.com
}

Submitted $: 10^{\text {th }}$ October 2019

Accepted : $20^{\text {th }}$ December 2019

Source of Support: None

Conflict of Interest: No

Citation: Pradhan M, Giri A, Rai D, Yadav R. Sjögren-Larsson syndrome: a case report of a rare neuro-cutaneous disorder. NMJ 2019;2 (2): 268-9. DOI 10.3126/nmj.v2i2.26866

\section{INTRODUCTION}

Sjögren-Larsson syndrome (SLS) is a rare autosomal recessive neuro-cutaneous disease featuring congenital ichthyotic hyperkeratosis, spastic diplegia and mild to moderate mental retardation. It occurs due to deficiency of microsomal fatty aldehyde dehydrogenase (FALDH) which results in the accumulation of fatty aldehydes and fatty alcohols in various tissues. This rare syndrome has mutations in ALDH3A2 located in chromosome 17p11.2. ${ }^{1}$ Neurological symptoms and signs appear during the first 2 years of life and consist of delay in reaching motor milestones due to spastic diplegia or much less commonly, of spastic tetraplegia. Here, we report a case presented with ichthyosis, bilateral lower limb spasticity, and mental retardation, diagnosed as Sjögren-Larsson syndrome.

\section{\# CASE REPORT}

A 12-year-male patient, born out of non-consanguineous marriage, presented to the Dermatology Department of Nobel Medical College, with generalized dryness of skin, inability to stand and walk along with poor speech. His birth history reveals delivery at pre-term (33 weeks of gestation) by normal vaginal delivery with a birth weight of approximately $2500 \mathrm{gm}$. Although there was no appearance of blister and erythema at any time since birth, his parents noticed generalized scaling since 2 months of age. On detailed history, his symptoms accompanied pruritus and progressive pattern of scaling. There was a delay in motor developmental milestones accompanied by conduction aphasia rendering him to withdraw socially. The similar history was not present in his siblings and other family members. 
Physical examination revealed generalized xerosis and scaling, most predominant around bilateral forearms and legs, including the face, with lichenification and accentuated skin markings (fig. 1,2). Palms and soles were spared. Teeth and nails were normal. There was a presence of moderate mental retardation. Genital examination revealed retractile testes. Spasticity and scissoring of the lower limb were present (fig.3). Knee and ankle deep tendon reflexes were exaggerated with extensor plantar response. Cranial nerve examination was unremarkable. A personal or family history of epilepsy was absent. Ophthalmological examination revealed bilateral horizontal nystagmus, severe dry eyes, primary open-angle glaucoma and fundoscopy revealed increased cup disc ratio.

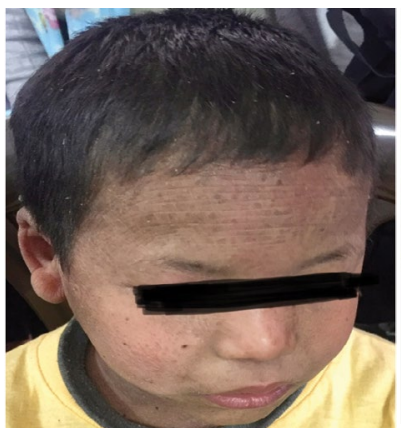

Figure 1: Ichthyosiform changes prominent over the forehead (Permission was obtained from the parents to publish the image)

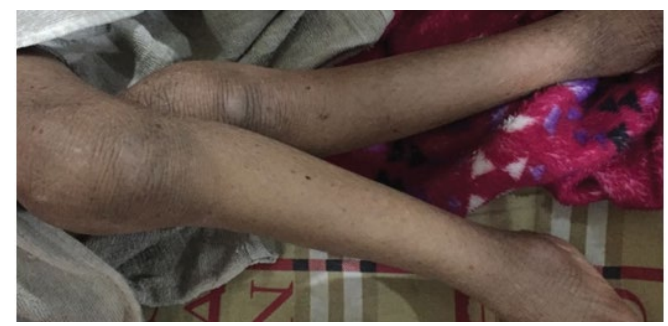

Figure 3: Spastic diplegia showing scissoring gait

Laboratory investigations that included complete blood count, renal function test, liver function test, thyroid function test, urinalysis, creatine kinase level were normal. With all the presenting findings, the patient was diagnosed as SjögrenLarsson syndrome.

\section{DISCUSSION}

In 1957, a rare syndrome consisting of congenital ichthyosis, spastic diplegia or tetraplegia (spastic pyramidal symptoms), aphasia associated with low-grade dementia was described by Sjögren and Larsson. ${ }^{2}$ The worldwide incidence of SjögrenLarsson syndrome (SLS) is 0.4 per 100,000 and Over 200 cases are reported across the globe. ${ }^{3}$

SLS was linked to fatty alcohol: NAD oxidoreductase (FAO) deficiency. FAO is a complex enzyme containing two different protein moieties which catalyze the oxidation process of fatty alcohol to fatty acids via fatty aldehyde. Further studies done in this field discovered Fatty Aldehyde Dehydrogenase (FALDH) activity in cultured fibroblasts from the patients with SLS signifying the importance of the FALDH component of FAO in SLS patients. FALDH, which is a microsomal enzyme, catalyzes the oxidation of medium and long-chain aliphatic aldehydes derived from numerous metabolisms in the body. ${ }^{4}$ More than 70 mutations in ALDH3A2 identified in patients suffering from SLS. ${ }^{5}$

Due to mutations, there is abnormal lipid accumulation in the membranes of the brain and skin, the combination of which is an important key for diagnosis. The clinical triad of generalized ichthyosis, mental retardation, and spastic paralysis is the main basis of diagnosis which was our case. Other relevant investigations carried out only for confirmation of the disease.

The pathognomic presence of glistening white dots in macula seen in one-third of cases, which may appear after several years of age. ${ }^{5}$ So, this finding is not present in all cases. MRI reveals diffuse symmetrical white matter hyperintensities, especially over periventricular frontal, parietal lobes, corpus callosum, and corona radiate, with sparing of subcortical white matter $U$ fibers. ${ }^{6}$ Due to insufficient resources, we report the case only on the basis of clinical findings.

Our case highlights the clinical triad of SLS. It is imperative to consider this rare, but important disease as a differential diagnosis when one deals with any case of ichthyosis. Moreover, family history is also quite important, rendering genetic counseling an option for such families. As skin involvement in this syndrome has greater magnitude, such patients likely go for dermatological consultation before neurological counsel, providing dermatologists an initial chance for proper diagnosis and management.

\section{CONCLUSION}

In any case presenting with ichthyosis, spastic diplegia, and mental retardation, it is important to consider SLS as a differential diagnosis though it is a rare disease.

\section{REFERENCES}

1. Pavithran K, Karunakaran M, Palit A et al. Disorders of Keratinization. In: Valia RG, Valia AR, editors. IADVL Textbook of dermatology 3rd ed. Mumbai: Bhalani Publishing House, 1002, (2008).

2. Sjögren T, Larsson T. Oligophrenia in combination with congenital ichthyosis and spastic disorders. Acta Psychiatr Scand. 1957;32:1108. Crossref

3. Carmana KB, Yimenicioglua S, Ekicia A, Yaku A. Sjogren-Larsson syndrome: A rare differential diagnosis of cerebral palsy. Int J Clin

Pediatr. 2012;1:133-5. doi: $\underline{\text { Crossref }}$

4. Rizzo WB. Sjögren-Larsson syndrome: molecular genetics and biochemical pathogenesis of fatty aldehyde dehydrogenase deficiency. Mol Genet Metab. 2007;90(1):1-9. Crossref

5. Gordon N. Sjogren -Larrson syndrome. Dev Med Child Neurol. 2007;49(2):152-4. Crossref

6. Dhanuka A K, Gupta M. Sjogren - Larsson Syndrome : a case report. Neurol India [serial online] 2002 [cited 2019 Dec 25];50:371. Available from: $\underline{\text { Crossref }}$ 